\title{
PROCESOS DE TRABAJO, NORMAS DE DE CALIDAD Y PARTICIPACIÓN SINDICAL EN LA PRODUCCIÓN INDUSTRIAL URUGUAYA.
}

\author{
WORK PROCESSES, QUALITY STANDARDS AND UNION \\ PARTICIPATION IN URUGUAYAN INDUSTRIAL PRODUCTION. \\ PROCESSUS DE TRAVAIL, NORMES DE QUALITE ET PARTICIPATION \\ SYNDICALE DANS LA PRODUCTION INDUSTRIELLE URUGUAYENNE. \\ PROCESSOS DE TRABALHO, PADRÕES DE QUALIDADE E \\ PARTICIPAÇÃO SINDICAL NA PRODUÇÃO INDUSTRIAL URUGUAIA. \\ Francisco Pucci* \\ Rodolfo Levin** \\ Nicolás Trajtenberg*** \\ Carlos Bianchi****
}

RESUMEN: El artículo está centrado en el estudio de las formas de participación laboral y de construcción de la legitimidad de las normas de calidad en diferentes empresas de la rama química y alimenticia en el Uruguay. En el trabajo se discuten los resultados del análisis cualitativo realizado a través de entrevistas en cuatro dimensiones i) la relación entre normas de calidad e innovación ii) la relación entre competencias y aprendizaje organizacional iii) la discusión en torno a los criterios de legitimidad de las normas de calidad y iv) la participación y cooperación de los trabajadores. Las conclusiones del trabajo son que las normas de calidad, si bien no modifican sustancialmente la matriz taylorista de producción, constituyen una forma de tecnificación del proceso de trabajo superior a

\footnotetext{
* Doctor en Sociología; Profesor Titular del Departamento de Sociología de la Facultad de Ciencias Sociales de la Universidad de la República (UDELAR), Montevideo, Uruguay; E-mail: francisco. pucci@cienciassociales.edu.uy

** Master en Sociología; Director del Instituto de Higiene, Facultad de Medicina de la Universidad de la República (UDELAR), Montevideo, Uruguay; E-mail: rolev@adinet.com.uy

*** Doctor en Sociología, Profesor Adjunto del Departamento de Sociología de la Facultad de Ciencias Sociales de la Universidad de la República (UDELAR), Montevideo, Uruguay; E-mail: nicolas.trajtenberg@cienciassociales.edu.uy

**** Doctor en Economía; Profesor Agregado del Departamento de Economía de la Facultad de Ciencias Económicas y Administración de la Universidad de la República (UDELAR), Montevideo, Uruguay; E-mail: cbianchi@iecon.ccee.edu.uy
} 
la implementada en las clásicas formas de organización del trabajo de las industrias sustitutivas de importaciones, promueven el desarrollo de criterios más universales de evaluación del trabajo y, en algunos casos, las posibilidades de instaurar procesos de re-taylorización que, de todos modos, constituyen formas más adecuadas que los modelos tradicionales de tipo paternalista.

Palavras clave: Competencias; Calidad; Participación; Cooperacion; Industria.

ABSTRACT: The article focuses on the study of forms of work participation and the construction of the legitimacy of quality standards in different companies of the chemical and food industry in Uruguay. In the work, the results of the qualitative analysis conducted through interviews in four dimensions are discussed i) the relationship between quality standards and innovation ii) the relationship between competencies and organizational learning iii) the discussion around the criteria of legitimacy of the quality standards; and iv) worker participation and cooperation. The conclusions of the work are that the quality standards, although they do not substantially modify the Taylorist production matrix, constitute a form of technification of the labor process superior to that implemented in the classic forms of work organization of the import substitution industries, promote the development of more universal criteria for the evaluation of work and, in some cases, the possibilities of introducing re-Taylorization processes that, in any case, constitute more adequate forms than traditional paternalistic models. .

Keywords: Competences; Quality; Participation; Cooperation; Industry.

RÉSUMÉ: L'article se concentre sur l'étude des formes de participation des travailleurs et la construction de la légitimité des normes de qualité dans différentes entreprises de l'industrie chimique et alimentaire en Uruguay. Dans le travail, seront présentés les résultats de l'analyse qualitative réalisée à travers des entretiens en quatre dimensions i) la relation entre normes de qualité et innovation ii) la relation entre les compétences et l'apprentissage organisationnel iii) la discussion autour des critères de légitimité des normes iv) la participation et la coopération des travailleurs. Les conclusions du travail sont que les normes de qualité, bien qu'elles ne modifient pas substantiellement la matrice de production taylorienne, constituent une forme de technification du processus de travail supérieure à celle mise en æuvre dans les formes classiques d'organisation du travail des industries de substitution aux importations, facilitent l'élaboration de critères plus universels pour l'évaluation du travail et, dans certains cas, la possibilité d'introduire des processus de re taylorisation les- 
quels, dans tous les cas, constituent des formes plus adéquates que les modèles paternalistes traditionnels.

Mots-Clés : Competences; Qualité; Participation; Coopération; Industrie.

RESUMO: $O$ artigo enfoca o estudo das formas de participação laboral e a construção da legitimidade das normas de qualidade em diferentes empresas da indústria química e alimentícia no Uruguai. No trabalho são discutidos os resultados da análise qualitativa realizada através de entrevistas em quatro dimensões i) a relação entre normas de qualidade e inovação ii) a relação entre competências e aprendizagem organizacional iii) a discussão em torno dos critérios de legitimidade da padrões de qualidade e iv) participação e cooperação dos trabalhadores. As conclusões do trabalho são que os padrões de qualidade, embora não modifiquem substancialmente a matriz de produção taylorista, constituem uma forma de tecnificação do processo de trabalho superior àquela implementada nas formas clássicas de organização do trabalho das indústrias de substituição de importações, promoven o desenvolvimento de critérios mais universais para a avaliação do trabalho e, em alguns casos, as possibilidades de introduzir processos de recaytalização que, em qualquer caso, constituem formas mais adequadas do que os modelos paternalistas tradicionais.

Palavras chave: Competências; Qualidade; Participação; Cooperação; Indústria.

\section{INTRODUCCION}

La noción de calidad ha variado significativamente en los últimos tiempos. Aplicado al trabajo y la producción este concepto ha tenido un particular impulso, orientándose hacia prácticas concretas de trabajo que reparan en la importancia de la promoción, desarrollo, control y aseguramiento de la calidad. En el transcurso del siglo XX la idea de calidad en la producción se transformó paulatinamente, desde de una perspectiva de «inspección» de la calidad del producto hacia otras concepciones más integrales como ser el «aseguramiento» y el «control de la calidad total». Estos nuevos enfoques conciben la calidad como un tópico que parte desde la alta gerencia de la empresa y alcanza a todos sus integrantes, al tiempo que apunta tanto hacia la calidad del producto como también a la de los procesos de gestión y producción. 
Uno de los motores del impulso de los Programas de calidad ha sido el desarrollo de mercados locales e internacionales con consumidores cada vez más exigentes y con creciente capacidad de imponer sus preferencias a la oferta. Esto ha contribuido a la implementación de sistemas de control de la calidad tanto de los productos que ingresan a un determinado mercado, como de los productos o insumos que una empresa adquiere de sus proveedores. La obtención de un patrón de calidad genéricamente aceptado cobra particular importancia en el comercio internacional, pudiendo convertirse en un requisito no arancelario a la entrada de productos en gran cantidad de países industrializados.

En este sentido, las pautas de calidad se incorporan como un criterio de competitividad entendida como capacidad competitiva de la empresa o del país, capacidad que no se basa tan sólo en la rebaja de costos o en la «competencia precio» sino en el desarrollo de productos o servicios diferenciados sobre la base de criterios de calidad. Para que esto sea posible es necesario el reconocimiento de esos criterios más allá de las fronteras nacionales o de divisiones corporativas, con lo cual toma particular relevancia el cumplimiento de normas técnicas de calidad que estén ampliamente aceptadas.

En el marco de estos procesos de cambio productivo, este artículo discute las competencias organizacionales necesarias para el sostenimiento de los programas de certificación de calidad en diferentes empresas de la rama química y alimenticia en el Uruguay, en base a una investigación financiada por la Comisión Sectorial de Investigación Científica de la Universidad de la República. Esta investigación se realizó entre los años 2005 y 2006, por lo que sus datos no están actualizados. Sin embargo, la presentación de la misma se justifica en la medida en que sus conclusiones reflejan tendencias estables en los procesos de transformación productiva del país, como lo muestran los trabajos de Quiñones, Supervielle y Acosta, (2015) y Quiñones, Segastini y Supervielle (2014)

En este trabajo discutiremos los resultados de la investigación realizada en tres dimensiones de análisis: i) la relación entre normas de calidad e innovación ii) la relación entre competencias y aprendi- 
zaje organizacional iii) la discusión en torno a los criterios de legitimidad de las normas de calidad y iv) La participación y cooperación de los trabajadores en los procesos de calidad.

La selección de las ramas no es arbitraria: interesa comparar dos industrias con trayectorias y composiciones diferentes. La industria química despliega una actividad intensiva en el empleo de capital, se encuentra en un mercado altamente competitivo y regulado a nivel internacional, y se caracteriza por dirigir la base de su producción al mercado interno. En cambio, la rama alimenticia es una de las industrias tradicionales de nuestro país, mantiene una participación importante en el rubro de exportables, y se caracteriza por una actividad intensiva en mano de obra. Las diferencias entre ambas ramas de actividad permiten establecer comparaciones de suma utilidad para el análisis de la construcción de competencias en tanto fenómeno propio de los procesos de mejora de calidad.

\section{1) METODOLOGÍA DE INVESTIGACIÓN.}

La investigación realizada desarrolló metodologías cualitativas para dar cuenta de las competencias movilizadas en los procesos de implementación de normas de calidad. Por las características señaladas en los antecedentes, estas competencias requieren un abordaje que permita comprender componentes cualitativos difícilmente cuantificables, como el conocimiento tácito, las relaciones de comunicación y las respuestas frente a situaciones de incertidumbre. Estas técnicas cualitativas de recolección de información permiten la emergencia de las representaciones sociales de los actores.

En este plano, la investigación realizó 20 entrevistas en profundidad al personal de diferentes categorías laborales en 2 empresas de la industria química y 2 empresas de la industria alimenticia, que implementaron las Normas ISO entre el 2002 y el 2005. Las mismas estaban orientadas al relevamiento de las variables cualitativas presentes en el diseño: conocimientos tácitos, rutinas laborales, relaciones de comunicación y confianza, procesos de traducción y actividad de regulación. La selección de las empresas no implica 
conformar una muestra significativa desde el punto de vista estadístico, sino que se procuró seleccionar, en lo posible, en las ramas consideradas, las empresas que tuvieran composiciones y trayectorias lo más disímiles posibles.

La investigación también implementó técnicas de observación directa en las empresas seleccionadas, a través de los cuales se analizaron los procesos de trabajo y las rutinas concretas que se implementan para dar cuenta de las incertidumbres técnicas que deben afrontar los recursos humanos en su contexto productivo. Esta técnica permite contrastar el discurso de los actores, recogido en las entrevistas, con las conductas concretas implementadas en las rutinas de trabajo.

Dada la gran variabilidad de situaciones de las distintas empresas, la caracterización del proceso de certificación de calidad en términos cualitativos sólo se puede realizar, a través de estudios de casos seleccionados a través de una muestra teórica de las principales tendencias que se encuentran en el universo. A continuación expondremos los principales resultados de las entrevistas realizadas a informantes calificados y a gerentes de recursos humanos de diferentes empresas de las ambas ramas (química y alimentos).

\section{2) NORMAS DE CALIDAD E INNOVACIÓN}

Las entrevistas realizadas a los organismos certificadores (LATU y UNIT en Uruguay) y a los auditores y asesores en calidad, muestran que los mismos atribuyen al proceso de certificación de calidad un carácter espontáneamente dinámico y esencialmente innovador. Esta perspectiva concuerda con el interés de estos actores de incorporar la mayor cantidad de empresas a procesos considerados innovadores y más eficientes. La calidad del producto se garantiza como el resultado de ciertas condiciones de realización bien identificadas. En este sentido, la noción de calidad que definen las normas ISO está fundada en la noción de aseguramiento, fundamentada en la hipótesis de que existe una estrecha relación entre el respeto de los compromisos asumidos por la empresa frente al cliente y la garantía de un tercero externo, que asegura la calidad del producto o del ser- 
vicio brindado. La certificación de calidad se plantea superar la evaluación de la calidad del producto por la evaluación de los procesos que conducen al producto final, procesos que pueden ser dinámicos $\mathrm{y}$ admitir diferentes formas.

La polémica a nivel académico se plantea de la siguiente manera: los procesos de certificación de calidad ¿introducen mecanismos de innovación en los procesos de trabajo, o refuerzan los componentes prescriptivos del modelo taylorista, formalizando procedimientos previamente diseñados? Los informantes calificados entrevistados consideran, en líneas generales, que los procesos de certificación de calidad introducen una nueva lógica de organización del trabajo y de racionalidad en los procesos productivos de las empresas. En este sentido, coinciden con la idea de que la formalización de los procesos no implican reforzar los elementos prescriptivos del proceso de trabajo, retomando la perspectiva de autores como Campinos y Marquette (1997), que plantean la necesidad de establecer fuertes diferencias entre el proceso de racionalización taylorista y el proceso de racionalización introducidos por los programas de calidad.

Las mismas tienen que ver con i) los objetivos y con ii) los procesos que se establecen. En relación a los objetivos, la base del dispositivo de calidad es asegurar al cliente la calidad de los productos que se verifica por la posibilidad de establecer su trazabilidad. La formalización de los procedimientos no se deduce de una descomposición racional de procesos de trabajo que en su origen fueron integrados, como en el caso del taylorismo, sino en la necesidad de establecer de manera escrita los procedimientos que se realizaron para lograr el producto. En este sentido, la formalización inherente a los procesos de certificación de calidad no necesariamente se opone a la innovación de la producción, como ocurre en los procesos de racionalización taylorista.

En relación a los procesos, las normas de calidad no se asocian a la implantación de normas organizacionales que prescriben la realización de las tareas, sino que los procesos de formalización generan modelos dinámicos, de experimentación colectiva, en los cuales la puesta a punto a través de la escritura de los procesos de trabajo consiste en una extracción-articulación de saberes que, si bien eran 
potencialmente formalizables, se mantenían de manera tácita. Las operaciones de develamiento involucran no sólo el saber tácito sino también el saber explícito o articulado a un nivel elemental.

Sin embargo, de las entrevistas realizadas en las empresas a los diferentes segmentos laborales no surge con claridad que la certificación de calidad tenga necesariamente en efecto innovador de los procesos de trabajo, aunque tampoco se pueda deducir que impliquen, en todos los casos, una mera formalización o burocratización de procesos que se mantienen según las pautas clásicas de producción que la empresa venía implementando. En algunos casos, la aplicación de las normas de certificación de calidad permitieron darle mayor eficiencia y transparencia al proceso de trabajo, lo que redundó en una mayor rentabilidad para la empresa. En otros casos, en cambio, se encontraron experiencias de innovación, que se desarrollan debido a que la empresa supo capitalizar los espacios de comunicación y de profesionalización del trabajo que se implementan en el marco de la certificación de calidad. La innovación no aparece asociada directamente a la implementación de las normas de calidad, sino que surge cuando, a las mismas, se le agregan condiciones de trabajo que permiten la movilización de competencias y conocimientos tácitos de los trabajadores.

Las entrevistas resaltan las diferencias entre los procedimientos escritos y las prácticas reales de los trabajadores, poniendo el acento en el proceso de reflexión colectiva como elemento clave para el desarrollo de la innovación. De todas maneras, la implementación de las normas de calidad en las empresas uruguayas parecen marcar el desarrollo de procesos de racionalización que tienen fundamentos diferentes a los de la racionalidad formal del modelo taylorista fordista. En ese sentido, es necesario marcar que los procesos de racionalización tayloristas tuvieron una implantación relativamente frágil en la industria uruguaya, por lo que las normas de certificación de calidad pueden aprovechar espacios de cooperación que persisten de los viejos modelos artesanales de producción, sin tener que establecer fuertes rupturas con modelos anteriores. 


\section{3) COMPETENCIAS Y APRENDIZAJE ORGANIZACIONAL.}

La racionalidad que imponen los procesos de certificación de calidad se sustentan en el desarrollo de competencias organizacionales que permiten tratar los fenómenos productivos como eventos que imponen desafíos que la empresa debe resolver. La literatura académica ha señalado la importancia del aprendizaje organizacional como mecanismo para establecer el vínculo entre la competencia de los trabajadores y la competencia de la empresa. La competencia de los asalariados se articula con el aprendizaje organizacional en diferentes dimensiones: a) como elemento de la dinámica del aprendizaje organizacional; b) como objeto de ese aprendizaje; c) y como capacidad para hacer evolucionar a la organización.

Para entender mejor este problema, se discriminan tres niveles de aprendizaje. El primer nivel es el individual, reconocido por la gestión de competencias. El aprendizaje organizacional pasa necesariamente por el aprendizaje de sus miembros. El segundo nivel de aprendizaje es el colectivo de trabajo, es decir, el grupo, la sección o la unidad de trabajo concreto donde se realiza el aprendizaje. En estos dos niveles, el aprendizaje genera mejoras locales, pero es débil en términos de mejora del desempeño global de la empresa. El tercer nivel de aprendizaje es el de la empresa. Se puede decir que el aprendizaje es un factor de eficiencia sólo si se sitúa en ese nivel. Esto supone que las reglas de funcionamiento de la organización son modificadas por el resultado de los aprendizajes colectivos. (Cavestro; Colin; Grasseur. 2002)

Otra diferenciación importante que podemos establecer es entre el conocimiento codificado y el conocimiento tácito. La tecnología incorpora un conjunto de conocimientos tácitos, presentes en la cabeza de los diferentes segmentos laborales (ingenieros, técnicos y obreros no calificados), que no pueden ser objeto de transferencia al mismo título que la parte material de la tecnología. Los problemas de transferencia de tecnología devienen por consecuencia un problema de traducción de un saber hacer tecnológico en un lenguaje comprensible por el utilizador. (Lambert, 1993). Como señala 
Arrow (1962), la tecnología no es un dato ni un conjunto de informaciones fácilmente apropiables. Integra saberes técnicos codificados, pero también un conjunto de conocimientos específicos que se ponen en marcha en las actividades productivas. Es más, si bien ciertos saber-hacer son susceptibles de ser trasmitidos por la vía de la demostración y del aprendizaje, una parte de estos, que son implicados en el desempeño de las actividades, son ignorados por los propios individuos y se mantienen a nivel tácito.

Precisamente, en el trabajo de campo, los responsables de recursos humanos de las empresas que se entrevistaron destacaron la articulación de saberes tácitos y codificados como punto clave de los procesos de certificación de calidad. Las entrevistas realizadas muestran también las innumerables tensiones entre la lógica de competencia y el aprendizaje organizacional. Esto implica diferenciar el modelo de competencia de las condiciones concretas de movilización de las competencias. (Cavestro; Colin; Grasseur. 2002) . Existen factores exógenos que establecen tensiones entre la lógica de competencia y los procesos de aprendizaje organizacional. La necesidad de adaptar este modelo a las realidades locales, o el carácter parcial o experimental que asume el mismo en una empresa son factores de tensión. Otra fuente de conflicto importante tiene que ver con el tiempo que insumen los procesos de aprendizaje. La adquisición de competencias requiere la implementación de procesos de intercambio y de acumulación de experiencias. Estos procesos se inscriben en una temporalidad que asegura la construcción de saberes y el desarrollo de nuevos comportamientos. El manejo de las temporalidades es un problema que han debido enfrentar las empresas entrevistadas para la implementación de los procesos de mejora de calidad. La dificultad de compatibilizar objetivos de rentabilidad a corto plazo con cambios en los procesos de trabajo que implican apuestas a largo plazo con resultados impredecibles, parece ser un punto de inflexión que diferencia el éxito o el fracaso de estos emprendimientos. El hecho de que las empresas que implementan procesos de mejora de calidad sean empresas grandes, asociadas a capitales extranjeros, les permite contar con recursos económicos que pueden atenuar las 
exigencias más urgentes de corto plazo, recursos que no poseen la mayoría de las empresas de capital nacional.

Las empresas que implementan procesos de mejora de calidad deben establecer mecanismos para reducir la incertidumbre que resulta de una apuesta a largo plazo en desmedro, muchas veces, de necesidades u objetivos de corto plazo. La movilización de la fuerza de trabajo a través del desarrollo de estrategias de participación de los trabajadores en la discusión de los temas de calidad de la empresa y el involucramiento de los mismos en los objetivos de la empresa, es uno de los mecanismos que las empresas entrevistadas han utilizado para generar relaciones de confianza que permitan reducir la incertidumbre organizacional. Un segundo mecanismo utilizado por las empresas es la apuesta a la re-calificación de la fuerza de trabajo, manteniendo, en la medida de lo posible, la estabilidad en el empleo de los trabajadores de mayor antigüedad en la empresa.

Sin embargo, en términos del proceso de trabajo, la creación de relaciones de confianza que permitan reducir las incertidumbres ligadas a la incompletitud del contrato de trabajo no se verifica en la mayor parte de las empresas estudiadas en este trabajo. Los componentes de confianza predominantes son de tipo adscripto, ligadas al origen social, al barrio o la posición en el proceso de trabajo, como expresión de formas de confianza identitaria. La sustitución de la confianza construida sobre la identidad de los oficios o de las relaciones de tipo comunitario por formas de confianza cognitiva, construidas sobre en las interacciones que se establecen entre oficios, profesiones y conocimientos diferentes, no parece un rasgo generalizado de los modelos de calidad implementados en el Uruguay. (Hardin, 1996) Las identidades construidas sobre la base de la separación entre trabajo intelectual y manual, entre trabajadores de planta y de oficina, entre profesionales y obreros no calificados, siguen marcando las lógicas de acción predominantes en el contexto industrial uruguayo.

Sobre la base de estas segmentaciones en los mercados internos de trabajo, los procesos de implementación de normas de calidad, en numerosas ocasiones, tienen el efecto contrario al deseado, en la medida en que refuerzan o acentúan estas divisiones establecidas pre- 
viamente, aumentando las distancias internas y profundizando los niveles de segmentación construidos históricamente. Esto significa que estos procesos permiten el desarrollo de competencias en sectores que previamente tenían altos niveles de calificación, y dejan de lado sectores de trabajadores menos calificados, que tienen previamente menor recursos cognitivos y emocionales para incorporarse exitosamente a estos procesos. De todas maneras, la profundización de la segmentación del mercado interno de trabajo tampoco se verifica en todas las empresas entrevistadas. En algunos casos, la implementación de procesos de calidad permitió el desarrollo de competencias en sectores poco calificados, o estableció espacios de comunicación entre trabajadores con diferentes niveles de calificación.

Las tensiones en la organización dependen en gran medida de la estructura interna y de los mecanismos de toma de decisiones. Existe una tensión esencial entre la lógica de adquisición y de reconocimiento de las competencias individuales y el funcionamiento de los colectivos de trabajo. La literatura académica ha mostrado como el reconocimiento de las competencias se traduce en una progresión individual de las carreras, acompañado muchas veces de incrementos salariales. Estas prácticas entran muchas veces en contradicción con una lógica colectiva de construcción de saberes en el seno de la organización. (Cavestro; Colin; Grasseur. 2002) Por un lado, hacen explícitas y visibles, en el seno de la organización y frente a las jerarquías, la contribución productiva individual. Por otra parte, la contribución productiva colectiva pasa a un segundo plano en el proceso de evaluación de los trabajadores. La lógica de la competencia acrecienta, en algunos casos, la competición inter-individual y reduce potencialmente la cooperación en el seno de los colectivos de trabajo. En otros casos conduce a disfuncionamientos importantes en la organización si las modalidades de cooperación son absorbidas por el reconocimiento de las performances individuales (Cavestro; Colin; Grasseur. 2002).

Las entrevistas realizadas indican que la individualización de las relaciones de trabajo no parece ser un problema significativo en las empresas que implementan procesos de mejora de calidad y de reestructuración de saberes. Esto se debe a diferentes razones. Por un 
lado, las entrevistas mostraron que el desarrollo de experiencias de gestión por competencias no se asocia directamente a los modelos de certificación de calidad. Si bien la aplicación de las normas de calidad implican, en algunos casos, mejoras en el proceso productivo, la gestión de recursos humanos se mantiene, en líneas generales, de acuerdo a los criterios tradicionales. Los mismos implican que se asignan a los trabajadores a determinados puestos de trabajo de acuerdo a la relación entre las exigencias del mismo y las calificaciones del individuo. Los criterios de promoción también mantienen pautas tradicionales de acuerdo a antigüedad, calificación y rendimiento.

Por otra parte, las normas de calidad se implementan en el marco de una negociación colectiva a nivel de rama de actividad que mantiene la inercia ligada al modelo de calificaciones, con una exhaustiva elaboración de categorías laborales según los criterios clásicos de formación y de antigüedad. Estos factores contribuyen a que se mantengan fuertes lazos de solidaridad y de acción colectiva en el seno de los grupos de trabajadores que participan en los sistemas de calidad, lo que mitiga las consecuencias que estos modelos han mostrado en otros contextos.

\section{4) LOS PROBLEMAS DE LEGITIMIDAD DE LAS NORMAS DE CALIDAD.}

La construcción de un sistema de calidad implica también un proceso de construcción de reglas, que se diferencian de las reglas típicas de los modelos tayloristas fordistas de organización del trabajo. Estas reglas se superponen a las viejas reglas que no desaparecen, o bien generan nuevas reglas de funcionamiento. En algunos casos, el modelo de competencia se instala sobre la organización existente. Las reglas se multiplican y se apilan para resolver los disfuncionamientos producidos por la gestión de competencias, generando procesos complejos de aprendizaje. En otras situaciones, emergen nuevas reglas de funcionamiento de la organización, volviendo caducas las anteriores. En general, las situaciones más comunes son las de transición entre un sistema y otro, sin rupturas radicales. (Cavestro; Colin; Grasseur. 2002) 
Las políticas de gestión de competencias llevan a una reformulación de los roles profesionales en cuatro direcciones: i) extienden considerablemente las obligaciones prescriptas para los obreros y los empleados ii) borran los principios anteriores de diferenciación jerárquica, mezclando estrechamente el contrato y la confianza según las formas de compromiso que antes solo correspondían a los directivos iii) atenúan el principio de diferenciación funcional entre concepción y ejecución. iv) transforman el rol de los cuadros medios que agregan a su clásico rol de control de la buena ejecución de los procedimientos un triple rol de negociación de objetivos, trasmisión de valores de la empresa y apreciación de la conformidad de los subordinados en todos estos planos. (Le Corre, 2003)

Reynaud (1988) ha distinguido, en los modelos clásicos de organización del trabajo, dos grandes tipos de reglas. Las primeras son las reglas organizacionales, definidas e impuestas por los mandos, que establecen de manera explícita, los mecanismos de control y de autoridad en la empresa. Junto a este sistema normativo, se desarrolla, en toda organización del trabajo, un conjunto de reglas autónomas, de carácter tácito, construido por los actores productivos, que permitan regular el trabajo real que se despliega en la unidad considerada. Este conjunto de reglas permite superar el conocido desfasaje entre el trabajo prescripto y el trabajo real, fenómeno típico ligado a los modelos tayloristas de organización del trabajo.

Analizando los procesos de innovación implementados en las empresas modernas, autores como Alter (1993) han señalado la existencia de una contradicción fundamental entre las lógicas de organización (standarización, coordinación y programación) y las lógicas de innovación (nuevas combinaciones, capacidad de reaccionar ante imprevistos, etc.) La des-taylorización representa, para este autor, un compromiso imperfecto entre dos lógicas sometidas a un ajuste constante: se trata de una ambivalencia organizacional que integra simultáneamente dos tipos de legitimidad en competencia: profesional, de un lado, y taylorista-racional, del otro. Este encuentro entre dos fuentes de legitimidad diferentes produce aprendizajes e híbridos organizacionales. 
En las organizaciones de producción de tipo taylorista, donde las reglas presiden formalmente a la acción, la relación salarial es de tipo contractual. Los dirigentes apuestan a la "capacidad de hacer" de los trabajadores y valorizan la misma. El rol de la confianza es mínimo porque las operaciones de juicio son confiadas, por encima de las relaciones interpersonales, a las instituciones de producción de conocimientos, o a organismos de control que intervienen posteriormente. (Le Corre, 2003) En cambio, las políticas de gestión de competencias ligadas a los sistemas de calidad producen reglas inestables y particularistas de naturaleza opuesta a las reglas burocráticas típicas de organizaciones tayloristas. Estos elementos refuerzan la incompletitud del contrato de trabajo, introduciendo obligaciones implícitas que no toman la forma de un reglamento sino de una obligación de asegurar la continuidad de la producción. Más que sustituir, agregan, a las obligaciones de medios que tienen los asalariados que ejecutan el trabajo, obligaciones de resultados, de creencias y de adhesión a los objetivos de la empresa. (Le Corre, 2003).

La dinámica de cooperación que introducen los procesos de calidad descansa, sobre la idea de que confianza y reglas formales o contratos no son dos modos alternativos sino complementarios de control. (Koenig, 1999) Los contratos son interpretados como reglas que permiten la interacción y la experiencia que, a su vez, permiten la emergencia de la confianza y de la cooperación. Las reglas son también el producto de relaciones comprometidas sobre la base de la reputación de los actores en juego o de una apuesta hacia el futuro. Las anticipaciones y el comportamiento de los que entablan la relación son continuamente evaluadas, y la confianza se interpreta como un proceso de aprendizaje. Confianza y contrato no son mutuamente excluyentes, sino que evolucionan y se interpenetran conjuntamente.

Las entrevistas realizadas parecen desmentir algunos de los argumentos desarrollados más arriba. La legitimidad de las normas de calidad no siempre se asocia al desarrollo de espacios de solidaridad y participación, si bien numerosas empresas implementan estrategias de este tipo. La legitimidad tampoco parece estar estrechamente asociada al desarrollo de espacios de confianza: muchos trabajadores, incluyen- 
do aquellos que pertenecen o representan a organizaciones sindicales, participan activamente de los procesos de certificación de calidad, al tiempo que mantienen una actitud crítica o desconfiada hacia las jerarquías empresariales, siguiendo el modelo de la participación paradójica desarrollada mostrada por Laville (1993).

En las empresas analizadas, la legitimidad de las normas de calidad parece provenir de su eficacia y de su creciente profesionalidad, lo que le otorga adhesión de los trabajadores, independientemente de haber participado o no en su construcción. Esta dimensión, señalada por Campinos y Marquette (1997), surge de la opinión de buena parte de los informantes calificados, de que la adhesión a las normas de calidad proviene del hecho de las mismas permiten que las empresas mejoren sus posibilidades en el mercado. Un ejemplo ilustrativo que corrobora esta tesis se puede buscar en el impacto que tuvo la crisis del año 2002 en la industria. Esta crisis implicó fuertes procesos de reestructura del personal, despidos y procesos de tercerización de numerosas empresas uruguayas. Para superar esta crisis, muchas empresas iniciaron procesos de certificación de calidad para lograr mejorar su posición en el mercado nacional e internacional. La adhesión de los trabajadores a estos procesos estuvo marcada, en numerosos casos, por la eficacia de las mismas en términos de resultados económicos y de la posibilidad de mantener los puestos de trabajo más que en la implementación de mecanismos de motivación y de participación.

\section{5) PARTICIPACIÓN Y COOPERACIÓN DE LOS TRABAJA- DORES.}

Linhart (1993) señaló como, en el caso de la industria francesa, se constatan numerosos esfuerzos por implicar a los trabajadores en la calidad de la producción, introduciendo una cooperación más horizontal entre los diferentes servicios y desarrollando proyectos multidisciplinarios. Desde el punto de vista de los resultados, la realidad parece estar lejos de los objetivos buscados. Los datos muestran un profundo desfasaje entre la amplitud de los esfuerzos realizados y la escasa envergadura de los cambios efectivamente implementados. 
En la industria francesa encontramos, siguiendo a este autor, tres tipos de realidades diferentes: las formas de organización del trabajo que rompen con el modelo anterior, las que refuerzan el taylorismo y las que, pese a los cambios realizados, reproducen totalmente el modelo que venían implementando. Esta situación parece similar a la que podemos encontrar en la industria uruguaya.

En los tres casos señalados, se asiste a una proliferación de fórmulas participativas como círculos de calidad, grupos de intercambio, etc. En el primer caso, el dispositivo de participación acompaña las innovaciones introducidas. En los otros dos casos, se trata en cambio de paliar las disfunciones generadas por el desfasaje entre una organización del trabajo rígida, enmarcada en preceptos tayloristas carentes de flexibilidad, y un entorno económico y tecnológico cada vez más turbulento y exigente. En todos los casos, el autor señala que el mayor problema es la construcción de confianza. Existe un déficit de confianza entre dirección y trabajadores, que viene de décadas de lucha de clases, antagonismos corporativos, división entre trabajadores de cuello blanco y cuello azul, etc. Esta desconfianza está ligada a la calidad de las interacciones pasadas y a la estructura asimétrica de poder que se dio históricamente entre los agentes, lo que afecta la reputación que cada uno tiene del otro.

Monchatre (2004), por su lado, defiende la idea de que los nuevos modelos productivos tienen su fundamento en una nueva calidad del trabajo marcada por el "automatismo social." sin implicar, más allá de las grandes transformaciones producidas, el desarrollo de relaciones sociales marcadas por la reconciliación y la confianza. La iniciativa del "management" ligado a los modelos de calidad, no frena la separación entre trabajador y trabajo, sino que se inscribe en un ideal de fluidez productiva y social. (Vatin, 1987) Se inscribe también en una serie de movimientos que contribuyen a desestabilizar las relaciones colectivamente construidas bajo el reinado de las calificaciones (Stroobants, 2003) expresada en la conversión de obreros en operadores.

Los procesos de gestión de competencias asociados a los modelos de certificación de calidad transforman los modos de producción con la introducción de una gestión de flujos y de numerosos automa- 
tismos, imponiendo nuevas exigencias los obreros no especializados de la cadena de montaje, en términos de polivalencia, cooperación y disponibilidad. Los cambios de talleres, el recurso a mano de obra joven y la delocalización de la producción en pequeñas unidades son los principales mecanismos de esta transición. Estas conversiones necesariamente impuestas, participan de una lógica de desmoralización del grupo obrero, que pasa por la desvalorización social de los antiguos obreros y la descalificación de las antiguas maneras de ser en nombre del modernismo. También participa de la conversión de obreros calificados en operarios. Constituye el medio para que la empresa defina sus propios oficios ajustando sus empleos al grado de fluctuaciones de la actividad, del mercado y de las estrategias productivas. (Monchatre, 2004) En este modelo, la construcción de formas colectivas de cooperación es sustituida por la tendencia a la individualización de la relación del operador con la empresa, el desarrollo de formas competitivas de relación social y la creciente rotación del personal al interior de la rama de producción. Este contexto no resulta favorable para la construcción de incentivos institucionales que recompensen los compromisos y la reputación de los agentes, los cuales, siguiendo a Hardin, (1996) favorecen el desarrollo de la confianza y de la cooperación entre los agentes productivos.

La posibilidad de constituir nuevas formas de pertenencia productiva depende estrechamente de la relación socio-histórica de los asalariados con la empresa, profundamente marcada por el contexto técnico-organizacional en el cual se ejerce el trabajo. (Laville, 1993) En las empresas marcadas por el modelo taylorista, las viejas características del trabajo no desaparecen en provecho de las nuevas exigencias. Estas se superponen a las antiguas y esta sedimentación se traduce en múltiples tensiones dentro del sistema productivo. La tentativa de conciliar estas exigencias contradictorias se manifiesta en las opciones que se toman, como la de pedir a los trabajadores que puedan llenar diferentes puestos con tareas parcelizadas más que construir una polivalencia a partir del enriquecimiento de las tareas. Esta contradicción se concreta también en un cierto número de oscilaciones y de compromisos inestables entre la especialización y la integración de 
servicios. Producto de estas tensiones, la adaptación del modelo taylorista revela estrategias paradójicas, en las cuales la utilización que se hace de la participación constituye un indicador. (Laville, 1993)

Las empresas se encuentran, entonces, con el dilema de crear las condiciones de una pertenencia productiva para involucrar al trabajador con los objetivos de calidad de la producción. En el modelo taylorista, la pertenencia productiva se expresa a través de una fuerte ambivalencia: la comunidad de referencia es una comunidad de productores, pero también una comunidad de oposición al orden establecido en la empresa. La búsqueda de la eficiencia se hace a través de un proceso de demarcación en relación a los modos de razonamiento impuestos. Se trata, según Laville, de un consentimiento paradójico o de una participación paradójica, término que busca dar cuenta de una actitud extremamente compleja que consiste en elaborar y movilizar saberes empíricos y capacidades de micro innovación, mejorando las condiciones y la eficacia de la producción, al tiempo que se expresa una desconfianza y una distancia con respecto a las reglas de juego de la empresa taylorista. (Laville, 1993) Estaríamos en este caso frente al caso en el cual existe cooperación pero no existe confianza entre las partes. Esta sería la modalidad de cooperación prevaleciente en el modelo taylorista fondista de producción.

En el caso uruguayo, las relaciones entre empresarios y trabajadores en la industria se mantienen, en su mayor parte, dentro de la lógica del modelo taylorista de producción y se expresan a través de formas de cooperación ambiguas, marcadas por la desconfianza en las reglas de juego tanto al interior de la empresa como en el marco más amplio de la negociación colectiva. El desarrollo de relaciones de cooperación que incluya altos componentes de confianza implica generar una estructura de incentivos a través de la cual los actores laborales puedan reconocer una comunalidad de intereses en que la misma funcione: mayor competitividad para el empresario, estabilidad en el empleo y mejores salarios para los trabajadores. Sin embargo, esta dimensión no alcanza. La construcción de relaciones de confianza requiere también que las interacciones entre empresarios y trabajadores modifiquen las representaciones que cada uno 
se hace del otro. Este aspecto, que releva de fuertes componentes de contingencia, puede explicar que, dada una misma estructura de incentivos, en algunas empresas sea exitosa y en otras fracase. Estos diferentes resultados muestran la diferencia entre asumir la perspectiva de la confianza relacional, cuyo fundamento es el sistema que se impone, y la perspectiva de la confianza social, cuyo fundamento está en la calidad de las interacciones de los agentes.

\section{CONCLUSIONES}

El análisis cualitativo mostrado reafirmó la idea de que la incorporación de las empresas uruguayas a los procesos de certificación de calidad es relativamente incipiente, y tiende a concentrarse en las grandes empresas multinacionales, que adoptan las formas de organización de sus casas matrices. Por otra parte, la motivación para incorporarse a estos programas pasa por las exigencias de calidad de mercados regionales o internacionales más que por las exigencias de calidad del mercado interno.

En el caso uruguayo, si bien encontramos diferentes situaciones en las empresas entrevistadas, los procesos de certificación de calidad parecen un espacio adecuado para el desarrollo de racionalidades cognitivas que pongan en juego el saber tácito de los actores, sus marcos de referencia profesional y sus capacidades de innovación. Las rutinas que se establecen en el marco de los programas de calidad no se oponen a la innovación o al mejoramiento de la calidad de los productos, sino que generan un conjunto de actividades que aumentan la reflexividad del proceso de trabajo. Este proceso genera una mayor profesionalización del trabajo obrero, incluso en los niveles menos calificados, lo que resulta una fuente de legitimidad importante para la aceptación de las normas de calidad.

Los procesos de calidad imponen una formalización de procesos sustentados en el concepto de trazabilidad, es decir, la posibilidad de reproducir los procesos que culminaron en la elaboración de determinado producto. Esta formalización ha permitido, en algunos casos, una producción más eficiente y programada de la producción, sin que ello haya 
introducido necesariamente procesos de innovación importantes. Tampoco parece haber una modificación sustantiva de la matriz taylorista de producción. La organización del trabajo sigue marcada por el concepto de puesto de trabajo y de categoría laboral de acuerdo a la clásica división técnica del trabajo de los modelos tayloristas de producción.

Sin embargo, pese a que esta matriz se mantiene inalterada en sus aspectos más sustantivos, los procesos de certificación de calidad han introducido algunos cambios importantes en las formas de organización del trabajo. En la mayoría de las empresas visitadas se verifican mejoras en los procesos de comunicación entre los trabajadores, mejor articulación entre el conocimiento técnico y el conocimiento práctico, y una mayor preocupación por la calidad del producto. En otros casos, el proceso de certificación de calidad parece ser una mera formalidad para obtener una vía de entrada a nuevos mercados. Sin embargo, incluso en estos casos, la imposición de controles y registros obligatorios en los diferentes pasos del proceso de trabajo han mejorado la eficiencia de la producción y generado nuevas oportunidades de calificación y de profesionalización para los trabajadores.

Los casos estudiados muestran que los procesos de certificación de calidad tienden a implementarse en empresas que tienen altos niveles de estabilidad de la mano de obra; muchas de ellas son empresas originadas en la etapa de sustitución de importaciones, que luego fueron adquiridas por capitales extranjeros, pero que mantienen una tradición "paternalista" y "familista" en relación a su personal. La antigüedad del personal no parece haber sido un obstáculo a la implementación de los procesos de certificación de calidad, contradiciendo las experiencias ocurridas en otros países. La literatura académica internacional ha resaltado la tensión que se introduce entre los viejos trabajadores ligados al modelo taylorista de trabajo, con dificultades para adaptarse a las nuevas formas de producción, y los trabajadores más jóvenes, más inclinados hacia estos cambios del trabajo, en los que encuentran mejores oportunidades de promoción y de desarrollo de sus carreras laborales. Estas tensiones no parecen estar presentes en las empresas analizadas en este proyecto. Esto puede ser explicado tentativamente por dos factores: en primer lugar, 
los cambios en la organización del trabajo no parecen alcanzar la envergadura de los que se implementan en contextos industriales más desarrollados. En segundo lugar, por las características socio-demográficas de nuestra sociedad, el mercado de trabajo uruguayo no posee el dinamismo existente en otros países. Esto implica que la estabilidad de la mano de obra sea una condición necesaria para mejorar los procesos de trabajo, habida cuenta la escasez de oferta de mano de obra calificada en algunas ramas de producción.

En las empresas visitadas, los procesos de certificación de calidad reciben la adhesión de los trabajadores y también de las organizaciones sindicales que los representan. En este sentido, es relevante hacerse la siguiente pregunta: iestos procesos tienen como condición la existencia de un sistema de relaciones laborales con altos niveles de acuerdo? O por el contrario, ¿son acaso los procesos de certificación de calidad los que generan una mejor relación entre empresarios y sindicatos? La respuesta parece orientarse en el primero de los sentidos. Las empresas que implementan procesos de certificación de calidad parecen tener una larga tradición de negociación y de acuerdos entre empresarios y trabajadores, más allá de conflictos puntuales. Otro aspecto relevante para comprender la actitud de los sindicatos en relación a los procesos de certificación de calidad tienen que ver con los cambios que ha experimentado el sindicalismo en los últimos años. La crisis de la industria y el aumento de las tasas de desempleo han generado un cambio importante en las estrategias generales del sindicalismo uruguayo. Las mismas pasaron de la defensa histórica de los niveles salariales a la preocupación por mantener las fuentes de trabajo como elemento prioritario de la acción sindical, particularmente en el sector industrial. Este cambio de la estrategia del movimiento sindical, que responde a las nuevas condiciones históricas en las cuales despliega su acción, es funcional a la implementación de cambios en la organización de la producción que mejoren la posición de la empresa en los mercados y su rentabilidad. En este contexto, la eficacia de las normas de calidad en términos de mejora de las capacidades productivas y de la colocación de la producción en mercados internacionales le otorga a las mismas una 
legitimidad de tipo práctico, originada en un aumento de la profesionalización individual y colectiva de la actividad laboral.

Los procesos de transformación de la industria uruguaya, al igual que otros contextos industriales latinoamericanos, se pueden explicar a través del concepto de "hibridación". (Novick et al; 2002) Esta perspectiva parte de la base de que existe una interacción compleja entre los modelos de organización del trabajo, las decisiones estratégicas de los núcleos empresariales o de las autoridades jerárquicas y los contextos nacionales e institucionales en los cuales se implementan estos procesos. El resultado es que las formas de organización del trabajo tienden a asumir formas "híbridas", en las cuales se yuxtaponen y se entremezclan componentes de diferentes modelos, sobre la base de la transformación o redefinición de los equivalentes funcionales del modelo de origen. En este plano, muchas de las nuevas formas de organización del trabajo implementadas en nuestros países no asumen la lógica integral de los nuevos modelos productivos, sino aspectos parciales y fragmentarios de los mismos. Otro componente importante de estos procesos es que, en numerosas ocasiones, el modelo original de trabajo tiene una fuerza emblemática que permea todas las futuras transformaciones que se dan en la organización de la empresa o del servicio.

Algunas empresas de la industria uruguaya en general, y de las ramas industriales seleccionadas, incorporan, de manera parcial e intersticial, componentes de calidad de los nuevos modelos productivos, pero sin llegar a constituir procesos de transformación profunda de los procesos de trabajo. Esta incorporación, a su vez, no se da en la gran mayoría de las empresas, que siguen produciendo de acuerdo de los formas clásicas de base taylorista que dieron origen a las formas de producción industrial modernas.

Los procesos de hibridación que se producen en los procesos de trabajo de las empresas entrevistadas refuerzan la tesis de que existe una continuidad y no una ruptura entre los conceptos de calificación y de competencias. En la medida en que no se han desarrollado en las empresas uruguayas modelos de gestión de competencias integrales, las exigencias de nuevas capacidades en el proceso de 
trabajo aparecen de forma intersticial, incorporadas en modelos de organización del trabajo donde la especialización y la tecnicidad del oficio siguen siendo prevalentes. Las formas de polivalencia que encontramos en las empresas entrevistadas son limitadas, cuando no son descalificantes. Por otra parte, las posibilidades de que las empresas capitalicen los conocimientos tácitos y las iniciativas de los trabajadores parecen condicionadas por una estructura que mantiene las segmentaciones clásicas entre trabajadores.

Si bien no podemos decir, a nuestro juicio, que los procesos de implementación de calidad sean simplemente formas de fluidez organizacional que permiten ajustar la fuerza de trabajo a los intereses de la empresa, o mecanismos a través de los cuales se disfrazan estrategias de reducción de costos y de ajuste de la cantidad de trabajadores a los requerimientos productivos, tampoco estamos frente a modelos que desarrollen formas de racionalidad comunicativa que transformen la relación del trabajador con su trabajo. El diagnóstico más ajustado parece ser el que ve en estos procesos una forma de tecnificación del proceso de trabajo superior a la implementada en las clásicas formas de organización del trabajo de las industrias sustitutivas de importaciones, el desarrollo de criterios más universales de evaluación del trabajo y, en algunos casos, las posibilidades de instaurar procesos de re-taylorización que, de todos modos, constituyen formas más adecuadas que los modelos tradicionales de tipo paternalista.

\section{REFERENCIAS}

ALTER, N. La crise structurelle des modèles d'organisation. Sociologie $d u$ Travail, Vol. 35, nº. 1, pp. 75-87, 1993.

ARROW, K. The economic implications of learning by doing. Revue of Economic Studies, Vol. 29, nº. 3, pp. 155-173, 1962.

CAMPINOS-DUBERNET, M.; MARQUETTE, Ch. Les normes d'assurance qualité iso 9.000: une opportunité de rationalisation des processus d'aprentissage de l'entreprise? Les cahiers de recherche GIP, n. 73,1997.

CAVESTRO, W.; COLIN, Th.; GRASSER, B. Compétences des salariés et compétence de la firme: une approche par l'apprentissage organisationnel. In: BROCHIER, D. (Dir.). La gestion de 
compétences: Acteurs et pratiques. Paris: Económica, 2002.

HARDIN, R. Trustworthiness. Ethics, Vol. 107, no. 1, pp. 26-42, 1996.

KOENIG, C. Confiance et contrat dans les alliances interentreprises. In: THUDEROZ, C. ; MANGEMATIN, V.; HARRISSON, D. (Dir.). La confiance. Approches économiques et sociologiques. Boucherville, Canadá: Éditeur Gaëtan Morin, 1999.

LAMBERT, G. Variables clés pour le transfert de technologie et le management de l'innovation. Revue Française de Gestion, n'. 94, pp. 49-72,1993.

LAVILLE, J. L. Participation des salariés et travail productif. Sociologie $d u$ Travail, Vol. 35, no. 1, pp. 27-47, 1993.

LE CORRE, S. Gestion des compétences et qualification du travail: une analyse des politiques de firme. In: DUPRAY, A.; GUITTON, C. ; MONCHATRE, S. (Dir.). Réfléchir la compétence. Approches sociologiques, juridiques, économiques, d'une pratique gestionnaire. Toulouse: Octarès Éditions, 2003.

LINHART, D. À propos du post-taylorisme. Sociologie du Travail. Vol. 35, $n^{\circ} .1$, pp. 63-74, 1993.

MONCHATRE, S. De l'ouvrier a l'opérateur: chronique d'une conversion. Revue française de sociologie, Vol. 45, nº. 1, pp. 69-102, 2004.

NOVICK; M; YOGUEL, G.; CATALANO, A.; ALBORNOZ, F. Adaptación de modelos productivos en países emergentes. El caso de la industria automotriz en la Argentina. Buenos Aires: LITTEC, UNGS, 2002.

QUIÑONES, M.; SEGANTINI, M.; SUPERVIELLE, M. Gestión de recursos humanos en la industria manufacturera exportadora de Uruguay,1991-2010. Montevideo: Ediciones Universitarias, CSIC, Colección Biblioteca Plural, 2014.

QUIÑONES, M.; SUPERVIELLE, M.; ACOSTA, M. J. Gestión de recursos humanos. Su configuración en el contexto de las lógicas empresariales uruguayas. In: El Uruguay desde la Sociología XIII. Montevideo, 2015.

REYNAUD, J. D. Les règles du jeu. L'action collective et la régulation sociale. Paris : Armand Colin, 1988.

STROOBANTS, M. L'individualisation des relations salariales par la gestion des compétences. In: DUPRAY, A.; GUITTON, C. ; MONCHATRE, S. (Dir.). Réfléchir la compétence. Approches sociologiques, juridiques, économiques, d'une pratique gestionnaire. Toulouse: Octarès Éditions, 2003.

VATIN, F. La fluidité industrielle. Essai sur la théorie de la production et sur le devenir du travail. Paris : Méridiens-Klincksiec, 1987. 